\title{
Perfil de calidad de vida, sobrepeso-obesidad y comportamiento sedentario en niños (as) escolares y jóvenes de secundaria guanacastecos
}

\section{Quality of Life Profile, Overweight-Obesity and Sedentary Behavior in Elementary and High School Children of Guanacaste}

\author{
Pedro Ureña Bonilla \\ Escuela Ciencias del Deporte de la Universidad Nacional \\ Heredia, Costa Rica \\ purena21@hotmail.com \\ Felipe Araya Ramírez ${ }^{2}$ \\ Escuela Ciencias del Deporte de la Universidad Nacional \\ Heredia, Costa Rica \\ fearaya1@hotmail.com \\ Braulio Sánchez Ureña ${ }^{3}$ \\ Escuela Ciencias del Deporte de la Universidad Nacional \\ Heredia, Costa Rica \\ brau09@hotmail.com \\ Jorge Salas Cabrera 4 \\ Escuela Ciencias del Deporte de la Universidad Nacional \\ Heredia, Costa Rica \\ josaca106@gmail.com \\ Luis Alberto Blanco Romero ${ }^{5}$ \\ Escuela Ciencias del Deporte de la Universidad Nacional \\ Heredia, Costa Rica \\ lblancoromero@hotmail.com
}

Recibido 02 de agosto de 2010 • Aceptado 31 de agosto de 2010

\footnotetext{
Doctor en Ciencias del Deporte con especialidad en Psicología Deportiva, por la Universidad de Deportes, Colonia, Alemania. Ha sido decano y vicedecano de la Facultad de Ciencias de la Salud de la Universidad Nacional, Costa Rica; así como también director interino de la Escuela Ciencias del Deporte de la Universidad Nacional, Costa Rica y del Programa de Maestría en Salud Integral y Movimiento Humano de la Universidad Nacional, Costa Rica.

2 Doctor en Fisiología del Ejercicio por la Universidad de Auburn. Académico de la Escuela Ciencias del Deporte de la Universidad Nacional, Costa Rica. Coordinador del Programa de Rehabilitación Cardiaca de la Escuela Ciencias del Deporte de la Universidad Nacional, Costa Rica Área de trabajo: La fisiología del ejercicio y la rehabilitación cardiaca.

3 Máster en Salud Integral y Movimiento Humano. Académico de la Escuela Ciencias del Deporte. Subdirector de dicha Unidad y coordinador del Programa de Ciencias del Ejercicio y la Salud. Áreas de trabajo: La fisiología del ejercicio y la prescripción de la actividad física para la salud.

4 Máster en Salud Integral y Movimiento Humano. Académico de la Escuela Ciencias del Deporte. Coordinador del Programa de Maestría en Salud Integral y Movimiento Humano de la Escuela Ciencias del Deporte. Áreas de trabajo: Fisiología del ejercicio y prescripción de la actividad física para la salud.

5 Máster en Salud Integral y Movimiento Humano. Académico de la Escuela Ciencias del Deporte. Áreas de trabajo: La metodología del entrenamiento y la prescripción de la actividad física para la salud.
} 
Resumen. El propósito de este estudio fue identificar el perfil de calidad de vida, sobrepeso-obesidad y comportamiento sedentario en un grupo de escolares y colegiales de la provincia de Guanacaste. Se contó con la participación de 635 estudiantes. Los (as) niños (as) y jóvenes participantes en el estudio cumplieron con un protocolo mediante el cual se les evaluó antropométricamente, también, completaron cuestionarios relacionados con el comportamiento sedentario y calidad de vida. En general, los resultados registrados mostraron una prevalencia del sobrepeso-obesidad del 13,9\%. Las actividades sedentarias más importantes fueron, en orden descendente, la pantalla chica (ver televisión, videojuegos, computadora), las actividades sociales y las culturales. El auto-reporte de calidad de vida mostró valores aceptables, aunque no superiores a 80 puntos en escala de 1 a 100. No se registraron relaciones significativas entre índice de calidad de vida global, sobrepeso-obesidad y comportamientos sedentarios, aunque algunos parámetros antropométricos como el porcentaje de grasa y el peso corporal mostraron correlaciones significativas con el comportamiento sedentario y con dominios específicos del constructo calidad de vida. El estudio brinda información valiosa en relación con aspectos centrales en el desarrollo del niño (a) o joven, no solo para autoridades en salud, sino también, para los directores (as) de centros educativos y, por supuesto, para padres y madres de familia.

Palabras clave. Perfiles, calidad de vida, sedentarismo, sobrepeso-obesidad.

Abstract. The purpose of this study was to identify the quality of life profile, overweight-obesity and sedentary behavior in a group of elementary and high school children of Guanacaste. 635 students participated in the study. The participants completed a protocol by which they were anthropometrically evaluated, and also filled up a questionnaire related to sedentary behavior and quality of life. In general, the findings reflected a prevalence of overweight and obesity of $13,9 \%$. The most important sedentary activities were, in descending order, the small screen (watching TV, video games, computer), and certain social and cultural activities. The self-reported quality of life index was within acceptable limits but not exceeding 80 points on a scale of 1-100. There was no significant relationship between the rate of the overall quality of life, overweight, obesity and some sedentary behaviors, although some anthropometric parameters like percentage of body fat and body weight showed significant correlation with sedentary behavior and specific aspects belonging to quality of life. The study provides valuable information to health authorities, directors of educational institutions and parents about key issues related to child development.

Key words. Profiles, quality of life, sedentary, overweight-obese.

\section{Introducción}

El sobrepeso, la obesidad y el comportamiento sedentario son temas centrales en la discusión relacionada con la calidad de vida, no solo porque están íntimamente ligados a las condiciones de salud de las personas, sino también, porque son factores relevantes en la definición de políticas vinculadas con la promoción de la salud. A pesar de que este es un tema relevante, en el contexto latinoamericano ha sido escasamente abordado, por lo que podría hablarse de un vacio investigativo importante.

El poco interés relacionado con el estudio de la temática supra-referida, especialmente en poblaciones sanas, hace que, a la fecha de hoy, todavía no se cuente con modelos conceptuales suficientemente fundamentados. En este sentido, el desarrollo de estudios epidemiológicos relacionados con la calidad de vida de las personas sanas puede brindar un aporte importante en la definición de políticas de salud pública, en la educación en general y en el desarrollo social de los pueblos.

El propósito de este estudio fue determinar los perfiles de calidad de vida, sobrepesoobesidad y comportamiento sedentario en niños (as) escolares y jóvenes colegiales de la Provincia de Guanacaste. Así como también, establecer relaciones y contrastes entre los distintos perfiles. 


\section{Referente teórico}

El constructo calidad de vida hace referencia a la forma particular en que las personas tienden a percibir y valorar su propia realidad (Quinceno y Vinaccia, 2008; Serra-Sutton, 2006). La calidad de vida se relaciona estrechamente con la habilidad de las personas para participar en actividades donde se vea involucrada la integridad bio-psico-social del ser humano (Serra-Sutton, 2006; Urzúa, Cortéz, Prieto, Vega y Tapia, 2009). En este contexto, el índice subjetivo de calidad de vida es un indicador válido de la salud de las personas, el cual permite valorar no solo la coherencia de las políticas de salud pública, sino también, la implementación de las mismas y su impacto en función de las necesidades sanitarias de la población (Oblitas, 2006; Ravens-Sieberer, Ellert y Erhart, 2007; Serra-Sutton, 2006).

A pesar de la importancia que tiene la percepción subjetiva del individuo en relación con la salud, el tema calidad de vida ha sido poco estudiado en grupos poblacionales de niños (as) y jóvenes "saludables" (Matsuda, Noguchi, Ameno y Kato, 2006; Ravens-Sieberer, Ellert y Erhart, 2007; Serra-Sutton, 2006; Urzúa, Cortés, Prieto, Vega y Tapia, 2009).

Hay una serie de dominios o factores que constituyen la realidad del niño (a) o joven, que son materia prima para la vivencia fenoménica, entre ellos: la familia, la escuela / colegio, el trabajo, los amigos, la salud (Quinceno y Vinnacia, 2008). La valoración subjetiva de cada uno de esos dominios permite obtener el índice global de calidad de vida o salud subjetiva (Montoya y Landero, 2008; Ravens-Sieberer, Ellert y Erhart, 2007).

Estudios realizados por Urzúa y otros (2009) reportan índices de calidad de vida similares entre hombres y mujeres, en la mayoría de los dominios en que son comparados. Sin embargo, en cuanto al "bienestar físico", los hombres muestran valores superiores a las mujeres; aunque en los dominios relacionados con el contacto social, las mujeres evidencian valores superiores.

En este mismo sentido, se reporta en niños (as) de 14 a 16 años diferencias significativas en las subescalas "bienestar físico" y "bienestar emocional" del constructo calidad de vida. Los niños comparados con las niñas reportan puntajes superiores (Rajmil y otros 2004; Urzúa y Mercado, 2008; Verdugo y Sabeh, 2002). En el caso de los adolescentes, no se reportan diferencias importantes entre hombres y mujeres, en cuanto a la valoración subjetiva de la calidad de vida (Modi, Loux, Bell, Harmon, Inge y Zeller, 2008).

En la población costarricense, los dominios mejor calificados en relación con la calidad de vida corresponden a la "familia", los "amigos" y la "salud" (Estado de la Nación en desarrollo humano sostenible, 2008). Esto significa que las vivencias de la población costarricense son valoradas positivamente en el contexto familiar, en el círculo de amigos y en relación con la percepción de la salud.

Estrechamente relacionado con la calidad de vida, el sobrepeso y la obesidad son condiciones orgánicas que afectan la salud objetiva y subjetiva de las personas (Kleiser, Schaffrath, Mensink, Prinz-Langenohl y Kurt, 2009; Quinceno y Vinaccia, 2008; Wang, Beydoun, Liang, Caballero y Kamanyuika, 2008; Wille, Erhart, Petersen y Ravens-Sieberer, 2008). Se trata de excesos patológicos de grasa corporal (Pérez, Sandoval, Schneider y Azula, 2008; Reilly, 2005), ocasionados por largos períodos de desequilibrio entre la energía que se consume y la que se gasta (Revuelta y Previnfad, 2005; Sweeting, 2008; Wilborn, Beckham, Campbell, Harvey, Galbreath y La Bounti, 2005). En el desarrollo de esta patología contribuyen, de manera decisiva, los ambientes obesogénicos, como por ejemplo, la sección de comidas en los centros comerciales, las sodas a nivel escolar - colegial e incluso el mismo hogar (Krieger, 2003; Pérez y otros, 2008; Revuelta y Previnfad, 2005). 
En relación con el sobrepeso y la obesidad, el panorama a nivel mundial es alarmante. En países como Alemania, aproximadamente el $15 \%$ de los (as) niños (as) y jóvenes entre 3 y 17 años son considerados con esa patología (Kleiser y otros, 2009; Wille y otros 2008). En otros países europeos como Grecia e Italia, se reportan índices de sobrepeso-obesidad superiores al 30\% (Briz, Cos, y Amate, 2007). Asimismo, en el estudio enKid realizado en España entre 1998 - 2000, en una población de niños (as) y jóvenes entre 2 y 22 años, se estimó una prevalencia de la obesidad del 13,9 \% (Hidalgo y otros, 2007). En niños mexicanos erradicados en Estados Unidos, se reporta una prevalencia de la obesidad del 7 \% (Modi y otros, 2008). En Estados Unidos para el 2000 la prevalencia de la obesidad en niños (as) de 6 a 11 años alcanzó el 15, 3\% y para adolescentes el 15,5\% (Hidalgo y otros, 2007; Wilborn y otros, 2005). Para el caso costarricense, se reportó en la encuesta de escolares 2004, promedios de sobrepeso y obesidad para niñas del 20,6 \% y para niños del 20,9 \% (Estado de la Nación en desarrollo humano sostenible , 2008).

Entre algunas de las consecuencias asociadas al sobrepeso y a la obesidad se mencionan la discriminación (Pérez y otros, 2008), la baja calidad de vida (Manonelles, Alcarz, Álvarez, Díaz, Luengo y Begoña, 2008) y los riesgos cardiovasculares (Kleiser y otros, 2009; Sweeting, 2008). A largo plazo, la obesidad infantil se relaciona con el aumento de la morbilidad (Modi y otros, 2008) y una mayor prevalencia de factores de riesgo cardiovasculares en la edad adulta (Hidalgo y otros, 2007; Reilly, Metheven, McDowel, Hacking, Alexander y Steward, 2003; Wille y otros, 2008).

Autores como Wille y otros (2008) han reportado relaciones negativas entre obesidad e índices de calidad de vida, especialmente en cuanto a la salud percibida, el bienestar psicológico y social. Entre las posibles explicaciones, se mencionan las eventuales estigmatizaciones y discriminación vinculada al sobrepeso y la obesidad, lo que puede afectar la autoestima de las personas, aumentar los niveles de ansiedad y deteriorar el estado anímico (Kolotkin y otros, 2006; Revuelta y Previnfad, 2005; Sweeting, 2008; Wang y otros, 2008).

El exceso de peso es más común en mujeres que en hombres (Yepez, Carrasco y Baldeón, 2008). Asimismo, las mujeres obesas comparadas con los hombres en condición similar, tienden a manifestar una menor calidad de vida (Modi y otros, 2008). En general, la mujer en condición de obesidad reporta un funcionamiento emocional más pobre que los hombres obesos (Galambos, Leadbeather y Barker, 2004). Es importante tener presente que, especialmente para el caso de las mujeres, ser delgadas es altamente valorado desde el punto de vista social, sobre todo, por cuanto la delgadez es considerada sinónimo de belleza (Sweeting, 2008).

Asimismo, los niños (as) con sobrepeso y obesidad comparados (as) con sus homólogos sin esta patología, muestran un deterioro mayor en el dominio amigos correspondiente al constructo calidad de vida (Wille y otros, 2008) y en el dominio salud física (Arif y Rohrer, 2006). En este mismo sentido, se reportan puntuaciones inferiores en salud física y calidad de vida general en niños obesos (García, Riaño, Somalo, Fernández y Málaga, 2005). En el caso de obesidad extrema, las mujeres comparadas con los hombres reportan valores significativamente más altos en la dimensión bienestar emocional; sin embargo, los hombres registran calificaciones superiores a las mujeres en bienestar físico y vida familiar (Modi y otros, 2008).

Por otro lado, el comportamiento sedentario se reporta como uno de los determinantes fundamentales del sobrepeso y la obesidad (Pérez y otros, 2008; Sweeting, 2008; Wilborn y otros, 2005; Buhring, Oliva y Bravo, 2009; Marshall, Biddle, Goreley, Cameron y Murdey, 2004). Por comportamiento sedentario se entiende toda aquella actividad que implica un gasto de energía menor a 1,5 mets (Buhring, Oliva y Bravo, 2009).

Entre los comportamientos sedentarios preferidos por los niños (as) y jóvenes se reportan: ver televisión, los videojuegos, las actividades sociales y educativas (Goreley, Marschall 
y Biddle, 2004). En general, se han registrado relaciones positivas entre comportamiento sedentario y obesidad (Lowry, Wechsler, Galuska, Fulton y Kann, 2002; Hidalgo y otros, 2007). Durante el período de la adolescencia es cuando se reporta una tendencia preocupante hacia la reducción de la actividad física (Buhring, Oliva y Bravo, 2009). Incluso en niños (as) se han observado niveles reducidos de actividad física extraescolar (Manonelles y otros, 2008), situación que agrava el problema del sobrepeso-obesidad y, consecuentemente, la calidad de vida. En este sentido, no cabe duda que el sobrepeso y la obesidad exacerban no solo la disfuncionalidad de las personas (Wilborn y otros. 2005) sino que también afectan la calidad de vida (Manomelles y otros, 2008).

El referente a nivel de países industrializados indica que, los (as) niños (as) y adolescentes ven televisión entre 2,5 y 3 horas diarias, y es esta la actividad sedentaria de mayor prevalencia (Goreley y otros, 2004). Es decir, estamos en presencia de un cambio epidemiológico en relación con el estilo de vida, donde está ganando terreno la preponderancia de elementos de entretención sustitutos de la actividad física (Buhring y otros, 2009; Revuelta y Previnfad, 2005). También, en niños (as) costarricenses, ver televisión se ha convertido en la actividad sedentaria en que se invierte la mayor cantidad de tiempo (Pérez y Smith, 2006).

La temática que se analiza representa una problemática en la que el comportamiento sedentario, el sobrepeso - obesidad y la percepción subjetiva de las personas se relacionan estrechamente y conforman una especie de círculo vicioso, lo que tiene serias implicaciones para el desarrollo social y humano de los pueblos, y obliga a emprender acciones inmediatas, especialmente en los grupos poblacionales de niños (as) y jóvenes (Kleiser y otros, 2009; Manonelles y otros, 2008; Pérez y otros, 2008; Wang y otros, 2008; Wille y otros, 2008).

\section{Metodología}

\section{Participantes}

Se contó con la participación de un grupo de 638 estudiantes pertenecientes a 8 de las instituciones educativas más grandes de la provincia de Guanacaste. La muestra estuvo conformada por 289 hombres y 349 mujeres. La edad promedio de los participantes fue de 13,03 años. Los grupos que participaron en el estudio fueron seleccionados por los directores (as) de cada institución educativa, según la disponibilidad de los mismos al momento del estudio. En todos los casos se contó con el consentimiento de los niños (as) y jóvenes para la realización de las distintas mediciones.

\section{Instrumentos y materiales}

El comportamiento sedentario fue medido mediante el cuestionario de actividades sedentarias (ASAQ). Este instrumento está compuesto por 11 actividades sedentarias típicas, las cuales son agrupadas en 4 categorías: Pantalla chica, educacionales, culturales y sociales. El procedimiento de respuesta consiste en anotar la cantidad de horas y minutos que se invierte diariamente en cada una de las actividades enlistadas en el instrumento. Se alcanzan correlaciones test retest superiores a 0,79 y coeficientes de correlación intraclase de 0,57 (Hardy, Booth y Okely, 2007).

La calidad de vida fue medida por medio del Kindl, instrumento desarrollado por Bullinger, von Mackensen y Kirchberger (1994) y traducido al castellano por Rajmil y otros (2004). El 
cuestionario contiene 24 preguntas distribuidas en 6 dominios: bienestar físico, bienestar emocional, autoestima, familia, amigos y escuela-colegio. Las respuestas se recogen en una escala likert de cinco categorías que oscilan desde $1=$ nunca, hasta $5=$ siempre. Las preguntas hacen referencia a la semana anterior. Asimismo, del perfil que entrega el instrumento se puede obtener un índice global de calidad de vida a partir de las medidas de las 6 dimensiones. Se obtiene una consistencia interna de 0,70 en el índice global, así como una estabilidad test retest en un rango que va desde 0,52 hasta 0,80 .

El registro de los valores antropométricos se realizó mediante la utilización de un tallímetro y de un monitor de grasa corporal marca Tanita (BF-683W), el cual se basa en el análisis de la impedancia bioeléctrica. El índice de masa corporal se calculó a partir de la fórmula IMC = peso / talla al cuadrado. Se utilizó como categorías de clasificación las sugeridas por Durstine, Moore, Painter y Roberts (2009).

\section{Procedimiento}

Se identificaron las principales instituciones educativas de primaria y secundaria de la provincia de Guanacaste (Cañas, Liberia, Nicoya y Santa Cruz), posteriormente se contactó a los directores (as) de dichos centros educativos, y se les explicó el propósito del estudio y se les solicitó el apoyo respectivo. La recolección de datos se realizó durante el mes de noviembre del 2009. Los cuestionarios fueron aplicados en forma grupal. Finalmente, se realizaron las mediciones antropométricas, se registró la estatura de cada participante, así como su peso y porcentaje de grasa.

\section{Análisis estadístico}

La información recolectada se procesó mediante el paquete estadístico para las ciencias sociales (SPSS) en su versión 15,0 para Windows. Los cálculos realizados incluyeron análisis de frecuencias, de medidas de tendencia central y de dispersión. El contraste de diferencias según sexo se realizó mediante la t-student para muestras independientes, cuando el supuesto de igualdad de varianzas se cumplió, en caso contrario se utilizó el U-test de Mann- Whitney. También se utilizó el coeficiente producto momento de Pearson. La aplicación de los análisis realizados fue precedida por la comprobación respectiva de los supuestos homogeneidad de varianza mediante el test de Levene y la normalidad de los datos, mediante gráficas y los valores correspondientes a la simetría y curtosis de la distribución. Para todos los efectos comparativos y de análisis de relaciones se consideró un nivel de significancia de 0,05 .

\section{Resultados}

En general, los jóvenes de colegio mostraron un peso y estatura significativamente superiores a los niños (as) de escuela. Sin embargo, en relación con el índice de masa corporal, los niños (as) de escuela obtuvieron valores significativamente inferiores. En cuanto al porcentaje de grasa no se encontraron diferencias estadísticamente significativas (cuadro 1). 
Cuadro 1

Valores promedio generales y diferenciados, según escuela-colegio para las variables antropométricas, calidad de vida y comportamiento sedentario

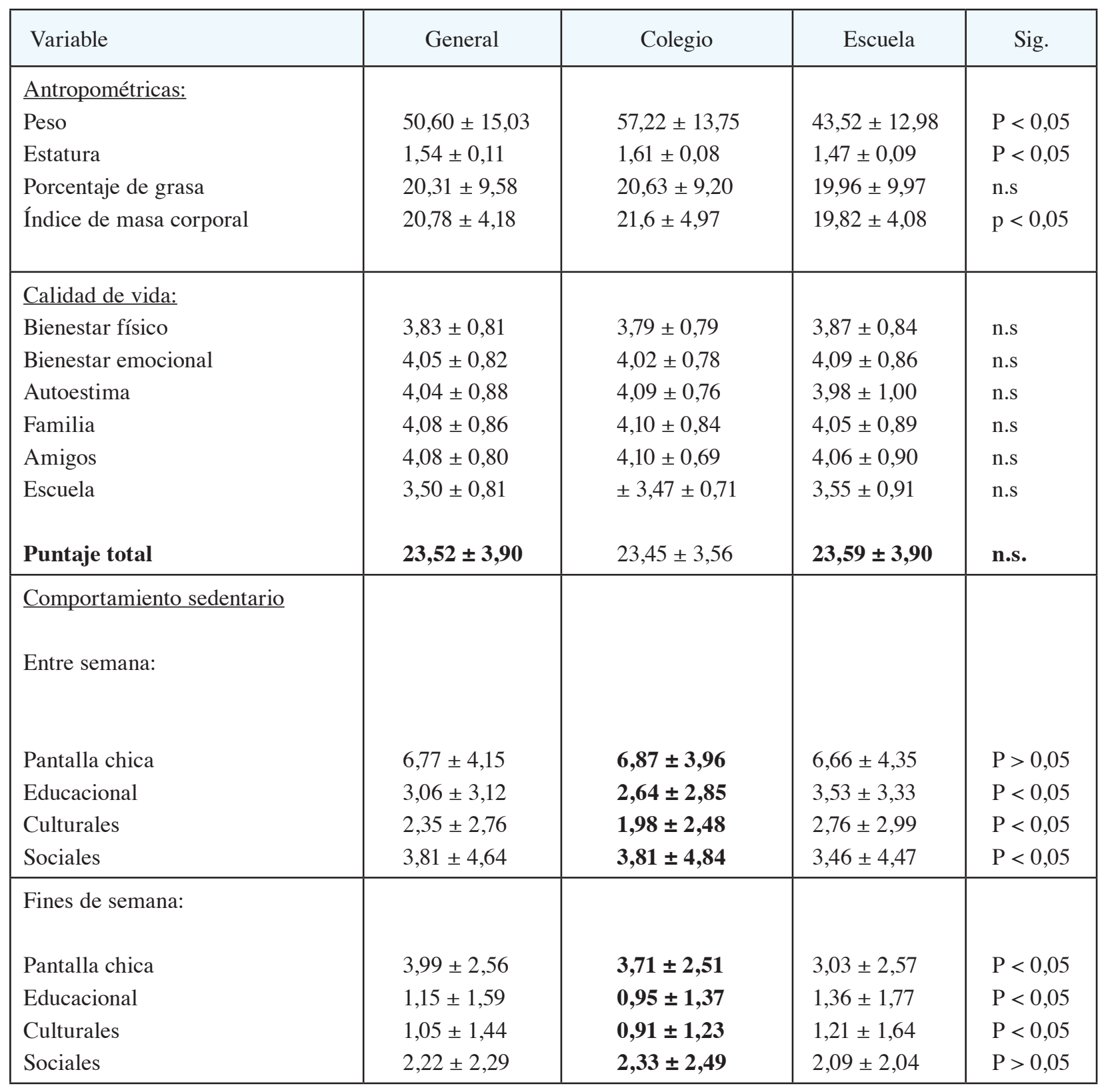

En relación con la calidad de vida, los valores generales muestran que los dominios mejor calificados corresponden a: "familia" y "amigos", asimismo, los peor calificados se refieren al "bienestar físico" y a la "escuela". No se registraron diferencias significativas entre los jóvenes colegiales y los niños (as) escolares. En ambos casos se mantuvo un comportamiento similar en cuanto a la calificación de los mejores y peores dominios. Es decir, los jóvenes de colegio al igual que los niños (as) de escuela le dan la mejor calificación a los dominios "familia" y "amigos" y la peor calificación a los factores "escuela" y "bienestar físico". 
El perfil de comportamiento sedentario entre semana mostró predominancia en actividades como: ver televisión, jugar en la computadora, y realizar tareas en la computadora (cuadro 1). El fin de semana se caracteriza, de manera particular, por el comportamiento sedentario "ver televisión". El contraste entre el comportamiento sedentario a nivel colegial y escolar arrojó diferencias significativas en la mayoría de los ítemes comparados. Entre semana, los niños (as) de escuela invierten significativamente más tiempo que los jóvenes colegiales en actividades educativas y culturales. Sin embargo, en cuanto a las actividades sociales, los jóvenes de colegio consumen significativamente más tiempo que los niños (as). Los fines de semana, el comportamiento de ambos grupos varía nuevamente. Los jóvenes de colegio, comparados con los niños (as) de escuela, gastan significativamente más tiempo ante la pantalla chica y en actividades sociales. Asimismo, los niños (as) de escuela, comparados con los jóvenes colegiales, consumen más tiempo en actividades relacionadas con aspectos educativos y culturales.

Cuadro 2

Porcentajes correspondientes al índice de masa corporal general y por sexo

\begin{tabular}{|l|c|c|c|}
\hline \multicolumn{1}{|c|}{ Categoría } & General & Hombres & Mujeres \\
\hline Bajo peso & $27,5 \%$ & $31,7 \%$ & $24 \%$ \\
Normal & $58,6 \%$ & $54 \%$ & $62,5 \%$ \\
Sobrepeso & $9,9 \%$ & $9,7 \%$ & $9,7 \%$ \\
Obesidad I & $3,3 \%$ & $3,5 \%$ & $3,8 \%$ \\
Obesidad II & $0,5 \%$ & $0,7 \%$ & - \\
Obesidad III & $0,2 \%$ & $0,3 \%$ & - \\
\hline
\end{tabular}

A pesar de que el mayor porcentaje de los niños (as) y jóvenes encuestados tienen un índice de masa corporal ubicado en la categoría normal, un porcentaje importante de los mismos arrojó valores correspondientes a la categoría bajo peso. Un 13, 9 \% mostró valores correspondientes a sobrepeso y obesidad.

El patrón de comportamiento en cuanto al índice de masa corporal, tanto para hombres como para mujeres, es similar al encontrado en la población general encuestada. En el caso de los hombres, se observa un aumento en el porcentaje correspondiente al índice de masa corporal normal, en detrimento del porcentaje correspondiente a la categoría normal. Un comportamiento inverso se observa en el caso de la población femenina, donde más bien se da un aumento en el porcentaje de personas que se ubican, según índice de masa corporal, en la categoría normal y una disminución del porcentaje de mujeres en la categoría bajo peso. Los porcentajes correspondientes a sobrepeso y obesidad I son similares entre hombres y mujeres. Finalmente, solo en el caso de los hombres se registró casos de obesidad II y III. 
Cuadro 3

Contraste de los valores promedio generales por sexo para las variables antropométricas, calidad de vida y comportamiento sedentario

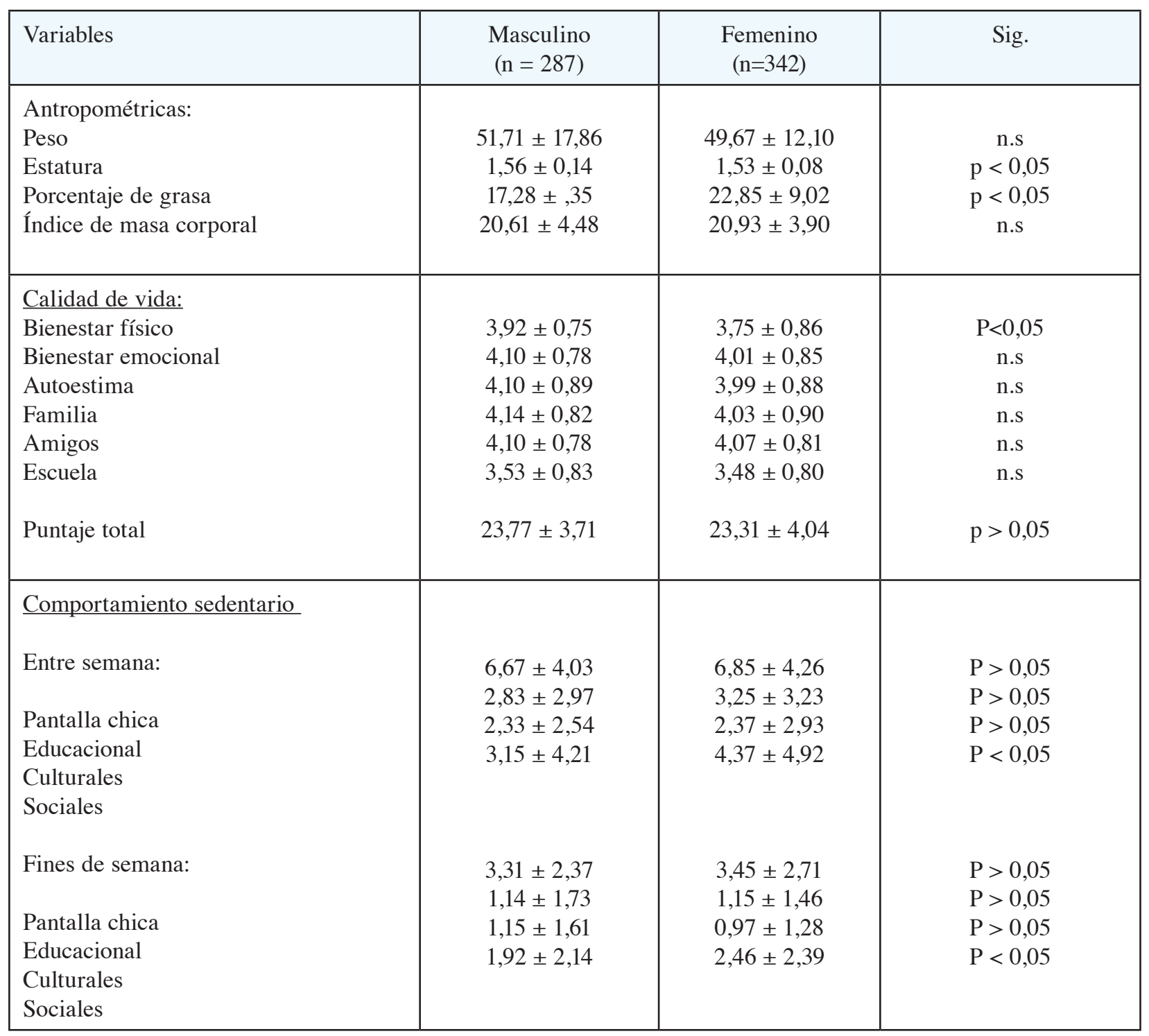

En relación con las variables antropométricas estudiadas, los resultados expuestos en el cuadro 2 muestran que los hombres, comparados con las mujeres tienen una estatura significativamente superior. Sin embargo, las mujeres muestran porcentajes de grasa superiores a los hombres.

En cuanto a la valoración de la calidad de vida, solo en el dominio "bienestar físico", los hombres muestran un puntaje significativamente superior a las mujeres; el resto de las dimensiones correspondientes a calidad de vida es similar. En general, el patrón de valoración de los distintos dominios indicadores de calidad de vida es similar en ambos grupos. Resulta importante señalar que los dominios peor calificados, tanto por hombres como por mujeres, corresponden al "bienestar físico" y a la "escuela".

La actividad sedentaria predilecta tanto para hombres como para mujeres atañe a la categoría "pantalla chica". Los datos registrados evidencian, además, que las mujeres comparadas con los hombres, invierten entre semana significativamente más tiempo hablando con los amigos (as). En 
contraste, los hombres comparados con las mujeres, pasan significativamente más tiempo viendo televisión y videos. En el resto de los ítemes evaluados, el comportamiento sedentario es muy similar. En relación con el fin de semana, las mujeres comparadas con los hombres, invierten más tiempo hablando con amigos (as) y asistiendo a la iglesia. El único comportamiento en donde los hombres invierten más tiempo que las mujeres, se refiere al ítem jugar o tocar algún instrumento musical.

En términos de asociación, el porcentaje de grasa correlacionó significativamente con los dominios autoestima $(r=-0,08 ; \mathrm{p} \leq 0,05)$, familia $(\mathrm{r}=-0,08 ; \mathrm{p} \leq 0,05)$ y con actividades sociales $(\mathrm{r}=-0,11 ; \mathrm{p} \leq 0,01$. Asimismo, el peso corporal correlacionó con actividades sociales $(\mathrm{r}=0,09 ; \mathrm{p} \leq$ $0,05)$ y con actividades educacionales $(\mathrm{r}=0,11 ; \mathrm{p} \leq 0,01)$. El índice de masa corporal correlacionó con las actividades sociales $(r=-0,09 ; \mathrm{p} \leq 0,05)$. El tiempo invertido en actividades sociales también correlacionó con el tiempo invertido en actividades educacionales ( $\mathrm{r}=0,09 ; \mathrm{p} \leq 0,05)$, actividades culturales $(r=0,14 ; p \leq 0,01)$ y con el tiempo ante la pantalla chica $(r=0,18 ; p \leq 0,01)$. A su vez, el tiempo ante la pantalla chica correlacionó con las actividades culturales $(r=0,14 ; \mathrm{p} \leq 0,01)$, familia $(\mathrm{r}=-0,08 ; \mathrm{p} \leq 0,05)$ y educacional $(\mathrm{r}=0,22 ; \mathrm{p} \leq 0,01)$. Finalmente, la actividad educacional correlacionó con los dominios escuela $(r=0,16 ; p \leq 0.01)$ y autoestima $(r=0,10 ; p \leq 0,01)$.

\section{Discusión}

El propósito de este estudio fue identificar perfiles en relación con la calidad de vida, comportamiento sedentario y sobrepeso-obesidad en niños (as) escolares y jóvenes colegiales de la provincia de Guanacaste. Los resultados encontrados mostraron índices de prevalencia del sobrepeso-obesidad inferiores a los reportados en el Informe del Estado de la Nación (Estado de la Nación en Desarrollo Humano Sostenible, 2008). Similar discrepancia se mantiene en relación con los datos publicados por Fernández y Moncada (2003). Asimismo, los datos registrados en el presente estudio, comparados con los referidos por Hidalgo y otros (2007), son considerablemente inferiores.

Aunque se trata de resultados relativamente alagüeños, es necesario estar atentos al comportamiento del sobrepeso y la obesidad en el grupo poblacional de niños (as) y jóvenes, especialmente por la gran cantidad de patologías asociadas a las mismas, cuyas consecuencias negativas son indiscutibles para la salud y calidad de vida de las personas (Kleiser y otros, 2009; Pérez y otros, 2008; Sweeting, 2008). Por otro lado, es claro que la identificación de índices de sobrepeso y obesidad en edades tempranas hace mucho más pertinente cualquier intervención que pretenda modificar los estilos de vida asociados a esta problemática (Kleiser y otros, 2009; Pérez y otros, 2008). Interviniendo en estas edades, se aumenta la probabilidad de que en la edad adulta, las personas tengan estilos de vida mucho más saludables.

El dato preocupante corresponde más bien a la alta prevalencia del bajo peso identificado, según la categorización propuesta por el Colegio Americano de Medicina Deportiva (Durstine y otros, 2009). Se trata de una situación que podría ser indicadora de niveles importantes de desnutrición que pueden atentar contra la salud de los niños (as) y jóvenes (Durstine y otros, 2009. En relación con este aspecto, existe suficiente evidencia en cuanto a las consecuencias que tiene la desnutrición para el desarrollo de los niños (as) y jóvenes (Leiva, Inzunza, Pérez, Castro, Jansana y Toro, 2001). Esto no solo desde el punto de vista de la salud física, sino también psicológica, lo que indudablemente puede afectar el rendimiento académico y la calidad de vida en general (Leiva, Inzunza, Pérez, Castro, Jansana y Toro, 2001). 
Es importante mencionar que los problemas de desnutrición afectan especialmente a los estratos poblacionales donde hay mayor privación socioeconómica, situación que se asocia, entre otras cosas, con deserción escolar y problemas de aprendizaje (Colquicocha, 2009; García, Riaño, Somalo, Fernández y Málaga, 2005; Leiva y otros, 2001). Los datos relativos a los índices de bajo peso encontrados en la población guanacasteca estudiada podrían estar asociados con situaciones socioeconómicas desventajosas importantes. De manera que tanto las autoridades en salud como en educación de la región deben estar atentos ante este tipo de problemática.

Por otro lado y en términos generales, el índice de calidad de vida registrado evidencia una valoración subjetiva aceptable y muy similar tanto para niños (as) a nivel de escuela, como para jóvenes a nivel de colegio. Juzgados en una escala de 1 a 100, la autovaloración que las personas encuestadas hacen de los dominios: bienestar físico, bienestar psicológico, autoestima, familia, amigos y escuela, alcanzó valores aceptables, aunque no superiores a 80. Resultados similares han sido reportados por Montoya y Landero (2008), Ravens-Sieberer y otros (2007), Rajmil y otros (2004) y Verdugo y Sabeh (2002). Asimismo, los datos recopilados corresponden con la evidencia reportada en el Estado de la Nación, en relación con el nivel de satisfacción con la vida en la población costarricense, donde los dominios mejor calificados corresponden a la relación con la familia y con los amigos (Estado de la Nación en desarrollo humano sostenible, 2008).

De manera particular, en relación con la calidad de vida, los dominios menos favorecidos en términos de la evaluación hecha por los jóvenes y niños (as), corresponde a la sensación de "bienestar físico" y "escuela". En el primero de los casos, la lectura de lo encontrado permite suponer que las personas encuestadas, probablemente, se han sentido enfermos (as), con molestias, cansados (as), o bien, con poca energía. Esta situación es muy propia de la vida escolar y colegial, especialmente por los niveles de estrés que se experimentan en ese contexto (Martínez y Díaz, 2007); o bien, por problemas normales de salud como resfríos u otros de naturaleza similar, los que también son frecuentes en este tipo de población.

En cuanto al dominio "escuela", se puede inferir niveles de disgusto de los (as) encuestados (as) en relación con su vida en el marco del escenario escolar y en razón de los deberes y obligaciones asociados a la escuela y colegio. Esto tiene que ver con la percepción de las tareas que se deben realizar, el gusto por las clases, el temor a sacar malas notas y la idea de tener que permanecer en el contexto escolar o colegial durante el tiempo que corresponde (Martínez y Díaz, 2007). Es válido afirmar que los niños (as) y jóvenes, tanto a nivel escolar como colegial, están sometidos a niveles importantes de tensión, situación que puede afectar desde su punto de vista la percepción de la calidad de vida. En este sentido, Martínez y Díaz (2007) reportan evidencias sobre una serie de factores psicosociales que pueden generar estrés a nivel escolar, entre los que mencionan, el trabajo académico, el rendimiento académico, la presión perturbadora y las evaluaciones. Aunado a los argumentos supra-referidos, Gómez y Jódar (2002) anotan el aburrimiento y la rebeldía como elementos consustanciales a la "experiencia institucional de ir a la escuela" (Martínez y Pérez, 2001). De manera que, no es extraño que los (as) niños (as) y jóvenes a nivel escolar y colegial, respectivamente, valoren de manera pobre el dominio correspondiente a la realidad escolar.

En cuanto a la diferenciación por sexo, los hombres, comparados con las mujeres, mostraron en general una mejor autovaloración en relación con su bienestar físico. Lo que permite suponer una mayor incidencia de problemas somáticos en la población femenina. En este sentido, Perarnau, Faluso, García y Doña (2007) argumentan que las mujeres preadolescentes y adolescentes, particularmente en los últimos años de escuela y a nivel colegial, tienen que lidiar con las dificultades que la menarquía acarrea, razón por la cual están más expuestas a sentirse incómodas, cansadas e indispuestas (Bocchino, 2004). Según estos autores, hay un patrón de síntomas emocionales, 
conductuales y orgánicos estrechamente relacionados con el periodo menstrual, lo que afecta la sensación de bienestar de las mujeres.

El perfil general encontrado en los dominios “bienestar psicológico", "autoestima”, "familia” y "amigos", se caracteriza por una buena valoración. Siguiendo la propuesta de Bullinger, Mackensen y Kirchberger, (1994), esto es indicador de que, a nivel de bienestar psicológico y autoestima, los (as) niños (as) y jóvenes encuestados, se sienten bien consigo mismos, pasan entretenidos, se divierten, se sienten orgullosos y están satisfechos con lo que hacen.

En relación con la familia y los amigos, el perfil reportado permite suponer ambientes familiares positivos, donde los (as) niños (as) y jóvenes parecen llevarse bien con los padres, se sienten a gusto en sus casas y están bien en relación con los límites que determinan los padres (Bullinger y otros, 1994).

Asimismo, en cuanto a los (as) amigos (as) el perfil general registrado es indicador de niveles de aceptación social adecuados, de buenas relaciones con los amigos (as) y de buenas oportunidades para intercambiar con amigos (as). De acuerdo con Díaz y otros, 2006) y en función de los resultados encontrados, se está hablando de una población de niños (as) y jóvenes, con buenos niveles de auto-aceptación, relaciones positivas, autonomía, dominio del entorno, propósito de vida y crecimiento personal. Esto significa que las personas del grupo encuestado, en términos generales, son conscientes de sus propias limitaciones y se sienten bien consigo mismas, además de que mantienen relaciones positivas con otras personas y conservan su propia individualidad en los diferentes contextos sociales en que interactúan. A todo lo antes mencionado, también se puede asociar la sensación de habilidad personal, lo que permite el dominio del entorno; así como el optimismo ante la vida; lo que está íntimamente relacionado con las posibilidades de crecimiento personal (Díaz y otros, 2006).

Desde el punto de vista social, se está en presencia de un subgrupo poblacional caracterizado entre otras cosas, por buenos niveles de integración y aceptación social (Blanco y Díaz, 2005). Es decir, se trata de personas "sanas" que se sienten parte de la sociedad y que tienden lazos sociales con la familia, amigos, vecinos, entre otros. Estos resultados coinciden plenamente con los reportados por el Estado de la Nación en Desarrollo Humano Sostenible (2008), particularmente en lo que corresponde a los dominios que se refieren a la relación con la familia y con los amigos. De hecho, estos parecen ser los factores definitorios del buen nivel de satisfacción de vida mostrado por los costarricenses en general.

Finalmente, el comportamiento sedentario predominante se asocia con el tiempo que los jóvenes y niños (as) pasan frente a la pantalla chica. Estos resultados son consistentes con lo reportado por (Goreley y otros, 2004; Pérez y Smith, 2006). Es decir, ver televisión, pasar frente a la computadora y los videojuegos son las actividades sedentarias predilectas por los niños (as) y jóvenes encuestados. Evidentemente, estamos en presencia de un comportamiento obesogénico (Sweeting, 2008) que no solo aumenta la probabilidad de problemas de salud en el orden de lo orgánico, sino también, en la valoración subjetiva de la misma (Pérez y otros, 2008).

Una prevalencia mucho menos importante se registra en cuanto a las actividades sedentarias relacionadas con el estudio y las de tipo social y cultural. A pesar de que tanto niños (as) como jóvenes coinciden en invertir cantidades de tiempo similares ante la pantalla chica, en relación con las actividades de tipo educativo y cultural, los niños (as) de escuela invierten mucho más tiempo que los jóvenes de colegio en dichas actividades. Esto podría explicarse por cuanto los niños (as) en edad escolar, acatan con más facilidad las indicaciones de los maestros (as) y padres de familia, en relación con la dedicación que tienen que darle a las actividades escolares (Bisegger, Cloetta, von Rueden, Abel, Ravens-Sieberer y The European Kidscreen Group, 2005). 
A nivel colegial, los jóvenes se encueBiseggertran en una situación de transición no sólo desde el punto de vista biológico-orgánico, sino también, emocional, donde la rebeldía frente a los deberes propios del colegio se manifiesta en un rechazo, en el que prefieren no invertir tiempo en actividades educativas (Gómez y Jódar, 2002). Otro aspecto que vale la pena mencionar tiene que ver con el hecho de que los jóvenes, a nivel de colegio, invierten mucho más tiempo que los niños (as) escolares en actividades sociales. También esta es una realidad asociada a la edad en que se encuentran los muchachos y muchachas en el colegio. Parte del desarrollo de su personalidad e identidad pasa por el tipo de vida social que logren desarrollar (Urzúa y otros, 2009). Todo parece indicar que, para los (as) jóvenes en esas edades, es fundamental ser aceptado socialmente, de manera que la búsqueda de amigos (as) y pasar tiempo con ellos es fundamental (Urzúa y otros, 2009). En consecuencia, no es extraño el hecho de que para ellos (as) las actividades sociales jueguen un rol fundamental.

Considerando la condición biológica sexo, los resultados encontrados mostraron también que las mujeres comparadas con los hombres invierten mayor cantidad de tiempo en actividades sociales. Por asuntos culturales y quizá biológicos, las mujeres, comparadas con los hombres, tienden más a socializar y a compartir; podría pensarse incluso que son más abiertas al intercambio (Urzúa y otros, 2009).

Los datos registrados mostraron también que cuanto mayor sea el índice de masa corporal, menor es la tendencia a involucrarse en actividades sociales. En este contexto, la hipótesis de la discriminación, producto de la forma corporal (Manonelles y otros, 2008; Pérez y otros, 2008), podría ser considerada como factor explicativo de dicho comportamiento. Consecuente con lo anterior, cuanto mayor es el porcentaje de grasa, menor es también el interés de las personas por involucrarse en actividades sociales. Estos datos coinciden nuevamente con la hipótesis de la discriminación producto del exceso de peso corporal.

Mientras mayor fue el porcentaje de grasa registrado, menores fueron también los niveles de autoestima y la valoración que se hace del ambiente familiar en relación con la calidad de vida. Esto coincide con lo reportado por (Revuelta y Previnfad, 2005; Wang y otros, 2008; Wille y otros, 2008). Todo indica que el sobre-peso y la obesidad hacen que las personas no se sientan tan bien consigo mismas; además de que afecta también la forma en que los niños (as) y jóvenes se llevan con los padres y la forma en que se sienten en su casa. No es de extrañar que, al incidir en la autoestima, se vean afectados otros dominios relacionados con la vida de los niños (as) y jóvenes (García y otros, 2005).

Paralelamente, cuanto mayor peso mostraron los (as) encuestados (as), mayor es la tendencia de los mismos (as) a involucrarse en actividades de tipo social y educativas. Asimismo, la tendencia a buscar relacionarse socialmente está muy relacionado con actividades educativas, culturales y con el tiempo invertido en la pantalla chica. Vale la pena destacar que los niños (as) y jóvenes que invierten grandes cantidades de tiempo viendo televisión, en videojuegos o en la computadora, tienden a no sentirse muy a gusto en casa.

Esto permite asignar a este tipo de actividades sedentarias un papel importante en el mundo de ocio del grupo poblacional encuestado (Aránguiz, 2005; Goreley y otros, 2004). Sin embargo, conforme los niños (as) y jóvenes pasan más tiempo ante la pantalla chica, también ocupan más tiempo realizando tareas escolares, estudiando y haciendo quehaceres propios de la escuela y colegio; así como también de tipo cultural. Como dato interesante, desde todo punto de vista, resulta el hecho de que la dedicación de los niños (as) y jóvenes a los quehaceres escolares y colegiales se relaciona, de manera positiva, con la valoración que se hace del ambiente escolar y de los deberes asociados al mismo. Finalmente, se evidenció que cuanto mejor se valore la auto-estima, más interés mostrarán los niños (as) y jóvenes por las actividades escolares. Definitivamente, una buena 
autoestima es factor determinante de una mayor capacidad de compromiso y responsabilidad (Cava, Musitu y Vera, 2000; Quinceno y Vinaccia, 2008) y de una mejor calidad de vida.

\section{Conclusiones}

Los resultados encontrados en el presente estudio evidencian la necesidad que existe de prestarle más atención a la vivencia fenomenológica del niño (a) y joven a nivel escolar y colegial. Esto significa considerar seriamente la auto-valoración subjetiva de las personas en relación con los distintos dominios relevantes en que interactúan. En este sentido, es importante conocer cómo se sienten los niños (as) y jóvenes en relación con la escuela y el colegio, con las tareas y deberes, con el ambiente social, con el hogar, consigo mismos. Todos estos son espacios de relación cuyo correlato fenoménico es fundamental como determinante de la valoración subjetiva de la calidad de vida.

Asimismo, es imperativo estar atentos al estilo de vida de los niños (as) y jóvenes, en especial porque, precisamente, en esas edades se sientan las bases del comportamiento que predominará en la adultez. Se debe tener la mira puesta en el diagnóstico y corrección de comportamientos obeso - génicos, de manera especial en el sedentarismo. Esto significa, entre otras cosas, ayudar al niño (a) y joven a organizar su tiempo libre, de manera que la actividad física pase a formar parte de su rutina de vida diaria. También significa hacer conciencia en el niño (a) y joven de los efectos negativos asociados a actividades sedentarias como: las largas horas frente al televisor, la computadora o los videojuegos.

Finalmente, es evidente que la promoción de estilos de vida físicamente activos se relaciona, de manera directa, con un gasto energético mayor, lo que reduce la probabilidad del sobrepeso y de la obesidad. Es decir, una vida activa físicamente no solo actúa en términos de promoción de la salud, sino también opera positivamente en la reducción del sobrepeso y la obesidad, y disminuye así el riesgo de patologías asociadas a la problemática referida y mejora a todo lo antes mencionado, la calidad de vida.

\section{Referencias bibliográficas}

Aránguiz, H. (2005). El sedentarismo, no es solo causa de la tecnologización. Revista Gestão Industrial, 1(1), 57-66.

Arif, A. y Rohrer, J. (2006, Junio). The relationship between obesity, hyperglycemia symptoms and health-related quality of life among Hispanic and non-Hispanic white children and adolescents [Relación entre la obesidad, síntomas de hiperglicemia y calidad de vida relacionada a la salud entre niños blancos hispanos y no hispanos y adolescentes]. BMC Family Practice, 7(3), 1-7. doi: 10.1186/1471-2296-7-3. Recuperado de http/www.biomedcentral.com/1471-2296/7/3.

Bisegger, C., Cloetta, B., von Rueden, U., Abel, T., Ravens-Sieberer, U. y The European Kidscreen Group. (2005). Health related quality of life: gender differences in childhood and adolescence [Calidad de vida relacionada a la salud: diferencias de género en la niñez y la adolescencia]. Soz. Praventivmed, 50(5), 281-291.

Blanco, A. y Díaz, D. (2005). El bienestar social: su concepto y medición. Psicothema, 17(4), 582-589. 
Bocchino, S. (2004, Julio). Salud mental de la mujer, síntomas y trastornos premenstruales. Clínica y tratamiento. Revista de Psiquiátrica del Uruguay, 68(1), 78-89.

Briz, F. J., Cos, A. I. y Amate, A. M. (2007). Prevalencia de la obesidad infantil en Ceuta. Estudio PONCE 2005. Nutrición Hospitalaria, 22(4), 471-477.

Buhring, K., Oliva, P. y Bravo, C. (2009, Marzo). Determinación no experimental de la conducta sedentaria en escolares. Revista Chilena de Nutrición, 36(1) 23-30. doi: 10.4067/S071775182009000100003

Bullinger, M., von Mackensen, S. y Kirchberger, I. (1994). KINDL - Ein Fragebogen zur Erfassung der gesundheitsbezogenen Lebensqualität von Kindern [KINDL - Cuestionario de calidad de vida relacionada con la salud en niños]. Sonderdruck Zeitschrift für Gesundheitspsychologie, 1, 64-77.

Cava, M. J., Musitu, G. y Vera, A. (2000). Efectos directos e indirectos de la autoestima en el ánimo depresivo. Revista Mexicana de Psicología, 17(2), 151-161.

Colomer, J. y Grupo Previnfad. (2005, Abril-Junio). Prevención de la obesidad infantil. Rev. Pediatr Atem Primaria, 7(26), 255-275.

Colquicocha, J. (2009). Relación entre el estado nutricional y rendimineto escolar en niños de 6 a 12 años de edad de la I. E. Tesis para obtener el título de Licenciada en Enfermería, Facultad de Medicina Humana. E.A.P. Enfermería. Universidad Nacional Mayor de San Marcos. Perú.

Díaz, D., Rodríguez, R., Blanco, A., Moreno, B., Gallardo, I., Valle, C. y otros. (2006). Adaptación española de las escalas de bienestar psicológico de Ryff. Psicotema, 18(3), 572-577.

Durstine, J. L., Moore, G. E., Painter, P. L. y Roberts, S. O. (2009). ACSM's Exercise management for Persons with Chronic Diseases and Disabilities [ACSM's Manejo del ejercicio para personas con enfermedades crónicas y discapacidades]. Champaign, IL.: Human Kinetics.

Estado de la Nación en desarrollo humano sostenible. (2008). Decimocuarto Informe: Bienestar Subjetivo en Costa Rica. FLACSO-México y OPAEP.

Fernández, A. y Moncada, J. (2003). Obesidad y sobrepeso en la población estudiantil costarricense entre los 8 y 17 años. Rev. Costarric. Cienc. Méd, 24 (3-4), 95-113.

Galambos, N. L., Leadbeater, B. J. y Barker, E. T. (2004, Enero). Gender differences and risk factors for depression in adolescence: a 4 year longitudinal study [Diferencias de género y factores de riesgo para la depresión en adolescentes]. Int J Behav Dev., 28, 16-25. doi: $10.1080 / 0165025034400023$ 
García M., Riaño, I., Somalo, L., Fernández, L. y Málaga, S. (2005). Calidad de vida relacionada con la salud en una muestra de niños y adolescentes obesos asturianos. Boletín Pediátrico, 45 (194), 277-289.

Gómez L. y Jódar, F. (2002, Otoño). Escuela, Aburrimiento y rebeldía. Athenea Digital, 2, 18-29. http://blues.uab.es/athenea/num2/Gomez.pdf

Goreley, T., Marshall, S. y Biddle, S. (2004). Couch Kids. Correlates of Television Viewing Among Youth [Correlatos entre ver televisión y la juventud]. International Journal of Behavioral Medicine, 11(3), 152-163.

Hardy, L., Booth M. y Okeley, A. (2007, Julio). The reliability of the Adolescent Sedentary Activity Questionnaire (ASAQ) Disabilities [Confiabilidad del cuestionario de actividades sedentarias en adolescentes]. Preventive Med, 45(1), 71-74.

Kleiser, C., Schaffrath, A., Mensink, G., Prinz-Langenohhl, R. y Kurth, B. (2009). Potential determinants of obesity among children and adolescents in Germany: results from the cross sectional KIGGS study [Determinantes potenciales de la obesidad entre niños y adolescentes en Alemania: resultados de un estudio transversal KIGGS]. BMC Public Health, 9(46), 1-14. doi:10.1186/1471-2458-9-46

Kolotkin, R., Zeller, M., Modi, A., Samsa, G., Polanichka, N., Yanovski. J. et al. (Marzo, 2006). Assessing Weight-Related Quality of Life in Adolescents [Valoración de la calidad de vida relacionada con el peso en adolescentes]. Obesity, 14(3), 448-457.

Krieger, N. (2003, Agosto). Genders, sexes, and health: what are the connections-and why does it matter? [Géneros, sexos y salud: cuales son las conecciones y porque estos es importante?] International Journal of Epidemiology, 32(4), 652-657. 10.1093/ije/dyg156

Leiva, B., Inzunza, N., Pérez, H., Castro, V. Jansana, J. M., Toro, T. et al. (2001). Algunas consideraciones sobre el impacto de la desnutrición en el desarrollo cerebral, inteligencia y rendimiento escolar. Archivos latinoamericanos de Nutrición, 51(1), 64-71.

Lowry, R., Wechsler, H., Galuska, D., Fulton, J. y Kann, L. (2002, Diciembre). Television Viewing and its Associations with Overweight, Sedentary Lifestyle, and Insufficient Consumption of Fruits and Vegetables Among US High School Students: Differences by Race, Ethnicity, and Gender [Ver televisión y su asociación con el sobrepeso, estilo de vida sedentario y consume insuficiente de frutas y vegetales en estudiantes de secundaria en US: diferencias según raza etnia y género]. Journal of School Health, 72(10), 413-421. DOI: 10.1111/j.1746-1561.2002. tb03551.x

Marshall, S. J., Biddle, S. J., Goreley, T., Cameron, N. y Murdey, I. (2004). Relationships between media use, body fatness and physical activity in children and youth: a meta-analysis [Relaciones entre el uso de los medios, la grasa corporal y la actividad física en niños y jóvenes: un meta-análisis]. International Journal of Obesity, 28(10), 1238-1246. doi:10.1038/ sj.ijo.0802706 
Manonelles, P., Alcarz, J., Álvarez, J., Jiménez, J., Luengo, E., Manuz, B. y otros (2008). La utilidad de la actividad física y de los hábitos adecuados de nutrición como medio de prevención de la obesidad en niños y adolescentes. Archivos de medicina del deporte, XXV(127), 333-253.

Martínez, B. y Pérez, O. (2001). Convivencia Escolar. Problemas y soluciones. Revista Complutense de Educación, 12(1), 295-318.

Martínez-Pérez, V. (2001). Convivencia escolar: problemas y soluciones. Revista Complutense de Educación, 12(1), 295-318.

Martínez, S. y Díaz, D. (2007). Una aproximación psicosocial al estrés escolar. Educación y Educadores, 10(2), 11-22.

Matsuda, T., Noguchi, M., Umeno, Y y Kato N. (2006, Noviembre). QOL research in child health. Present state and issues [Investigación en calidad de vida en la salud de los niños. Situación actual y tópicos]. Japanese Journal of Public Health, 53, 805-817.

Modi, A., Loux, T., Bell, S., Harmon, C., Inge, T. y Zeller, M. (2008). Weight-specific Health-related Quality of Life in Adolescents with Extreme Obesity [Salud relacionada a la calidad de vida específica al peso en adolescentes con obesidad extrema]. Obesity a reserch journal, 16(10), 2266-2271. doi:10.1038/oby.2008.347

Montoya, B. y Landero, R. (2008). Satisfacción con la vida y autoestima en jóvenes de familias monoparentales y biparentales. Psicología y Salud, 18(1), 117-122.

Oblitas, L. (2006). Psicología de la salud y calidad de vida. México: Thompson.

Perarnau, M. P; Fasulo, S. V; García, A. y Doña, R. (2007). Síndrome premenstrual y trastorno disfórico premestrual en estudiantes universitarias adolescentes. Fundamentos en Humanidades, Año VIII, 16(2), 153-163.

Pérez, E. C., Sandoval, M. J., Schneider, S. E. y Azula, L. A. (2008, Marzo). Epidemiología del sobrepeso y la obesidad en niños y adolescentes. Revista de Posgrado de la Vía Cátedra de Medicina, 179, 16-20.

Pérez, R. y Smith, V. (2006). Uso de medios en niños y niñas de escuela públicas y privadas de San José. Revista Ciencias Sociales Universidad de Costa Rical, I-II (111-112), 131-142.

Quinceno, J. M. y Vinaccia, S. (2008). Calidad de vida relacionada con la salud infantil: una aproximación conceptual. Psicología y Salud, 18(1), 37-44.

Rajmil, L., Serra-Sutton, V., Fernández, J., Berra, S., Aymerich, M., Cieza, A., et Al. (2004). Versión española del cuestionario alemán de calidad de vida relacionada con la salud en población infantil y de adolescentes: el Kindl. Anales de Pediatr 60(6), 514-521. 
Ravens-Sieberer, U. Ellert, U. y Erhart, M. (2007). Gesundheitsbezogene Lebensqualität von Kindern und Jugendlichen in Deutschland. Berlin: Springer Medizin Verlag.

Reilly, J. J., Metheven, E., McDowel, Z. C., Hacking, B., Alexander, D., Steward, L. y otros (2003, Setiembre). Health consequences of obesity [Consecuencias en la salud de la obesidad]. Archive of Disease in Childhood, 88(9), 748-752.

Reilly, J. J. (2005, Setiembre). Descriptive epidemiology and health consequences of childhood obesity [Epidemiología descriptiva y consecuencias obesidad en la niñez sobre la salud]. Best. Practice \& Research Clinical Endocrinology \& Metabolism, 19(3), 327-341.

Serra-Sutton, V. (2006). Desarrollo de la versión española del Child Health and Illness Profile para medir el estado de salud percibido en la adolescencia. (Tesis doctoral). Universidad Autónoma de Barcelona, Barcelona.

Sweeting, H. (2008). Gendered dimensions of obesity in childhood and adolescence [Las dimensiones del género en la obesidad en la niñez y la adolescencia]. Nutrition Journal, 7 (1), 1-14. doi:10.1186/1475-2891-7-1

Urzúa, A., Cortés, E., Prieto, L., Vega, S. y Tapia, K. (2009, Junio). Autoreporte de la calidad de vida en niños y adolescentes escolarizados. Revista Chilena de Pediatría, 80(3), 238-244.

Urzúa, A. y Mercado, G. (2008, Julio). La evaluación de la calidad de vida de los y las adolescentes a través del KIDDO - Kindl. Terapia psicológica, 26(1), 133-141.

Verdugo, M. y Sabeh, E. (2002). Evaluación de la percepción de calidad de vida en la infancia. Psicothema 14(1), 86-91.

Wang, Y., Beydoun, M., Liang, L., Caballero, B. y Kumanyuika, S. (2008). Will All Americans Become Overweight or Obese? Estimating the Progression and Cost of the US Obesity Epidemic. [Llegarán a tener sobrepeso y obesidad todos los americanos? Estimación de la progresión y el costo de la epidemia de la obesidad en USA]. Obesity a research journal, 16(10), 2323-2330. doi:10.1038/oby.2008.351

Wilborn, C, Beckham, J., Campbell, B., Harvey, T., Galbreath, M., La Bounty, P., et al. (2005). Obesity: Prevalence, Theories, Medical Consequences, Management, and Research Directions [Obesidad: prevalencia, teorías y consecuencias médicas, manejos y líneas de investigación]. Journal of International Society of Sports Nutrition 2(2), 4-31. 10.1186/1550-2783-2-2-4

Wille, N., Erhart, M., Petersen, C. y Ravens-Sieberer, U. (2008, Diciembre). The Impact of Overweight and Obesity on Health-Related Quality of Life in Childhood-Results From an Intervention Study [El impacto del sobrepeso y la obesidad sobre la calidad de vida relacionada a la salud tomados de los resultados de un estudio de intervención en la niñez]. BMC Public Health, 8(421). doi:10.1186/1471-2458-8-421

Yepez, R., Carrasco, F. y Baldeón, M. (2008). Prevalencia de sobrepeso y obesidad en estudiantes adolescentes ecuatorianos del área urbana. Archivos latinoamericanos de Nutrición, 58(2), 139-143. 